Short Research Paper

2011; 8(4):283-286

\title{
Minimally Invasive Parathyroidectomy Ising Surgical Sonography
}

\author{
Karim W Sadik, Malcolm Kell, Tom Gorey ${ }^{凶}$ \\ Mater Misericordiae University Hospital, Dublin 2, Ireland
}

$\triangle$ Corresponding author: Prof Thomas F Gorey, MCh FRCSI FACS, Mater University Hospital, Dublin 7. Ireland. Tel -353 1 830 0345, tgorey@mater.ie

(C) Ivyspring International Publisher. This is an open-access article distributed under the terms of the Creative Commons License (http://creativecommons.org/ licenses/by-nc-nd/3.0/). Reproduction is permitted for personal, noncommercial use, provided that the article is in whole, unmodified, and properly cited.

Received: 2010.10.30; Accepted: 2011.04.12; Published: 2011.04.15

\begin{abstract}
Minimally invasive parathyroidectomy is the procedure of choice for primary hyperparathyroidism due to parathyroid adenoma. Localization of the offending adenoma in minimally invasive parathyroidectomy (MIP) has been described in the literature aided by isotope, telescope or ultrasound guidance. We present a prospective study of two techniques based on surgeon experience. Thirty patients diagnosed with primary hyperparathyroidism at the Mater hospital in Dublin, Ireland were randomized to have a minimally invasive parathyroidectomy using surgical sonography (MIPUSS) or the conventional unilateral open procedure (OP) over a two year period. The age, sex and serum calcium/parathormone were comparable in both groups. There was no significant difference in complications between the two groups with temporary hypocalcemia occurring in 3 patients undergoing unilateral neck exploration and in 2 MIPUSS patients. There was one transient episode of recurrent laryngeal neuropraxia occurring in the OP group which resolved at 30 day follow-up. The incision size, operating time, hospital stay, and required post-operative analgesia were all markedly reduced in the MIPUSS group. In conclusion, MIPUSS is safe, effective and has advantages in terms of operating time, incision size and early discharge.
\end{abstract}

Key words: Minimally invasive parathyroidectomy, surgical sonography

\section{Introduction}

Primary hyperparathyroidism (pHPT) is a common endocrine disorder, which can affect 1 in 700 people. In $80 \%$ of cases the cause is a solitary parathyroid adenoma. Recently the use of perioperative $99 \mathrm{mTc}$-sestamibi with intraoperative localization has led to the development of minimally invasive parathyroidectomy (MIP) [1,2]. This technique facilitates unilateral neck dissection with less surgical trauma and shortens hospital stay compared with four-gland exploration. However, MIP requires an intraoperative gamma probe, which necessitates either scheduled 99mTc-sestamibi injection on the day of surgery with preoperative scanning and intraoperative measure- ment of gamma emission or $99 \mathrm{mTc}$-sestamibi injection on different days: one preoperative scan and another for intraoperative gamma emission. This may not only complicate scheduling for surgery but also exposes patients and medical personnel to an unnecessary dose of radioactivity. Ultrasound provides an excellent modality for visualizing structures in the neck [3]. Up to $90 \%$ of parathyroid adenomas can be visualized with ultrasound. We previously suggested intraoperative ultrasonograpy as a viable localizing modality [4]. Herein we confirm that intraoperative ultrasonography can be used to complement or replace standard imaging for the localization of para- 
thyroid adenomas.

\section{Methods}

\section{Patient Selection}

We studied all patients presenting with a biochemical diagnosis of primary hyperparathyroidism between July 2003 and May 2005. Twenty patients underwent MIPUSS and 10 patients were selected for OP. We excluded one patient with four gland hyperplasia on Sestamibi and ultrasonographic studies.

\section{Preoperative Management}

All thirty selected patients underwent pre-admission investigative imaging using $99 \mathrm{~m}$ Tc-sestamibi. Injection of 20 to $25 \mathrm{mCi}$ 99mTc-sestamibi was performed and views were acquired at 15, 60, and 180 minutes utilizing identical acquisition parameters. A consultant radiologist and surgeon reviewed all scans.

\section{Operative Procedure}

Following informed consent, patients underwent general anesthesia with endotracheal intubation. Patients were positioned supine with slight head up tilt and the neck extended in a head ring.

\section{MIPUSS Group}

Once positioned, a surgeon trained in ultrasonography used a $10 \mathrm{MHz}$ linear array ultrasound probe (Sonosite, USA) to localize the lesion. The adenoma was identified as a hypoechoic area close to the thyroid. The site was localized percutaneously and the neck marked over the maximum transverse and longitudinal planes. Where these two lines intersected a $3 \mathrm{~cm}$ transverse mark was placed on the neck. Following skin preparation, the area of incision was infiltrated with $10 \mathrm{cc}$ of local anesthetic (xylocaine $0.5 \%$ with 1:10,000 adrenaline) and the incision made. Subplatysmal planes were created and the strap muscles were mobilized. The thyroid plane was then entered between the strap muscles and the sternocleidomastoid muscle. The plane was then continued down to the adenoma. Once visualized, the adenoma was not immediately mobilized, instead a 14-gauge needle was placed through the wound onto the adenoma. Once the lesion was concordant with ultrasound findings and the recurrent laryngeal nerve identified and avoided, the adenoma was then excised and confirmed on frozen section. The neck was closed with interrupted absorbable sutures and interrupted non-absorbable sutures to the skin which were re- moved at 48 hours and replaced with adhesive strips. No drain was used.

\section{OP Group}

No ultrasound was used intraoperatively in these cases. After administration of general anesthesia and intubation the patient was similarly positioned as above. A $6 \mathrm{~cm}$ unilateral incision was made in order to allow exploration of superior and inferior parathyroids on the side localized by preoperative sestamibi scan. The anatomic approach and closure are as described above.

\section{Postoperative Management}

All patients were examined by the surgical housestaff on a daily basis for wound hematomas as well as signs of hypocalcemia. Analgesia was administered in the form of intravenous morphine. Diet was slowly re-introduced and serum calcium levels were checked on postoperative day 1 . All patients were discharged on oral pain medication. Following discharge, patient incision, calcium and PTH levels were reviewed in the outpatient setting on postoperative day 30.

\section{Results}

All patients in the MIPUSS group had single adenomas; in 2 cases the incision was extended as the adenoma was difficult to identify. On table ultrasonography was successful at localizing a solitary adenoma in each of the MIPUSS group. Preoperative Sestamibi scan only successfully localized the adenoma in $58 \%$ of the patients in this same group and in $70 \%$ of the patients in the open group. The average operative time was 45.21 minutes for MIPUSS and 66 minutes for OP $(p<0.05)$. The average size of incision in the MIPUSS group was $2.86 \mathrm{~cm}$ versus $6.1 \mathrm{~cm}$ in the OP group. No MIPUSS patients showed evidence of recurrent laryngeal nerve injury, however one OP patient had transient neuropraxia which resolved at 30 day followup. On average the MIPUSS group required $51.4 \%$ less intravenous analgesia than the OP group. All but 2 of the MIPUSS patients were discharged on the $1^{\text {st }}$ post-operative day compared to only one of the OP patients. At 30 day follow-up all patients were well and asymptomatic. Calcium levels had returned to normal and no patients required supplementation. Formal histology confirmed parathyroid adenoma in all cases. 
Table I. OP vs. MIPUSS comparison

\begin{tabular}{|c|c|c|c|}
\hline & Open Procedure (10) & MIPUSS (20) & P Value \\
\hline Average Age (years) & $61.5+/-10.46$ & $65.0+/-14.59$ & $\mathrm{P}=0.52$ \\
\hline M:F Ratio & $3: 7$ & $5: 15$ & \\
\hline Average pre-op total serum calcium level (mmol/L) & $2.90+/-0.35$ & $2.96+/-0.26$ & $\mathrm{P}=0.83$ \\
\hline Average Parathyroid hormone level Pre-op (pg/mL) & $197.5+/-167.47$ & $226.29+/-246.63$ & $\mathrm{P}=0.75$ \\
\hline Average duration of Procedure (minutes) & $66+/-20.52$ & $45.21+/-9.13$ & $\mathrm{P}<0.05$ \\
\hline Average incision size (centimeters) & $6.1+/-0.57$ & $2.86+/-0.66$ & $\mathrm{P}<0.05$ \\
\hline Average duration of in-hospital stay (hours) & $47.5+/-9.81$ & $22.64+/-4.13$ & $\mathrm{P}<0.05$ \\
\hline $\begin{array}{l}\text { Average amount of intravenous morphine used prior to discharge } \\
(\mathrm{mg})\end{array}$ & $10.05+/-1.77$ & $5.25+/-1.12$ & $\mathrm{P}<0.05$ \\
\hline Average postoperative total serum Calcium level (mmol/L) & $2.31+/-0.29$ & $2.29+/-0.08$ & $\mathrm{P}=0.83$ \\
\hline Number of patients with temporary Hypocalcemia & 3 & 2 & \\
\hline Number of patients with RLN Paresis & 1 (temporary) & 0 & \\
\hline Patients in whom we Extended the Incision & 0 & 2 & \\
\hline
\end{tabular}

\section{Discussion}

In this series, ultrasound has been shown to be a useful imaging adjunct to preoperative 99mTc-sestamibi scanning. It has successfully identified parathyroid adenomas without the need for intraoperative gamma emission. The shorter hospital stay and the significant decrease in analgesic requirements are probably related to the difference in incisional size and tissue mobilization between the two groups. Training surgeons to utilize ultrasound intra-operatively requires a small time commitment. It also allows the surgeon to function independent of radiology staff and further time constraints. Furthermore, it is a cost saving imaging modality when compared with sestamibi injections and the subsequent imaging.

In this study we have shown MIPUSS to be a simple and accurate on-table technique that specifically localizes the offending adenoma while achieving the smallest incision possible. We believe this is a useful technique in the majority of patients with parathyroid adenomas, however it is unlikely to be as helpful in patients with multigland hyperplasia or ectopic parathyroid gland adenomas. Given the size of incision and decreased analgesia we envision that
MIPUSS could potentially be a day-case procedure performed under local anesthesia.

\section{Conclusion}

MIPUSS was successful in 18 of 20 patients. MIPUSS is a safe and effective means of treating hyperparathyroidism through a small incision. This approach allows limited dissection and early discharge for the majority of patients and avoids the use of intra-operative nuclear localization and its associated problems.

\section{Conflict of Interest}

The authors have declared that no conflict of interest exists.

\section{References}

1. McGreal G, Winter DC, Sookhai S, Evoy D, Ryan M, O'Sullivan GC, Redmond HP. Minimally invasive, radioguided surgery for pHPT. Ann Surg Oncol 2001; 8(10): 856-60.

2. Goldstein RE, Billheimer D, Martin WH, Richards K. Sestamibi Scanning and Minimally invasive radioguided parathyroidectomy without intraoperative parathyroid hormone measurement. Ann Surg 2003; 237(5): 722-31.

3. Light VL, McHenry CR, Jarjoura D, Sodee DB, Miron SD. Prospective comparison of dual-phase technetium-99m-sestamibi scintigraphy and high resolution ultrasonography in the evaluation of abnormal parathyroid glands. Am Surg 1996; 62(7):562-7. 
4. Kell MR, Sweeney KJ, Moran CJ, Flanagan F, Kerin MJ, Gorey TF. Minimally invasive parathyroidectomy with operative ultrasound localization of the adenoma. Surg Endosc 2004; 18:1097-1098. 